\title{
Hartmagnetische Schichten für hochpräzise Mikroskopie
}

\author{
Die Anforderungen an die optische Auflösung von Mikroskopen und die Präzision von \\ Mikroskoptischen sind enorm. Hartmagnetische Schichten des Fraunhofer IST tragen dazu bei, \\ kleinste Zellstrukturen sehr schnell und genau zu erfassen.
}

Mikroskop- und Labortechnik sind ein unverzichtbares Hilfsmittel im Kampf gegen Viren und Bakterien. Sie unterstützen Wissenschaftler bei der Suche nach Impfstoffen und Therapien, etwa gegen SARS-CoV-2. Am Fraunhofer-Institut für Schcht und Oberflächentechnik IST in Braunschweig entwickeln Forscher hartmagnetische Kobalt-Samarium(CoSM)-Schichten für magnetische Maßstäbe. Diese Bänder werden in den Mikroskoptischen der Firma Dr. ITK Kassen eingesetzt. Im Zusammenspiel mit Sensoren und einem Auswert-Algorithmus erhöhen sie die Positioniergenauigkeit des Mikroskoptischs, auf dem die Probe zur Beobachtung abgelegt wird. Ein weiterer Vorteil: Mikroskoptische, die mit der magnetischen Positionierung arbeiten, lassen sich sehr kompakt bauen.

Das Team rund um Dr. Ralf Bandorf bringt die CoSm-Schichten auf unmagnetische Metallbänder auf. So erhalten diese eine

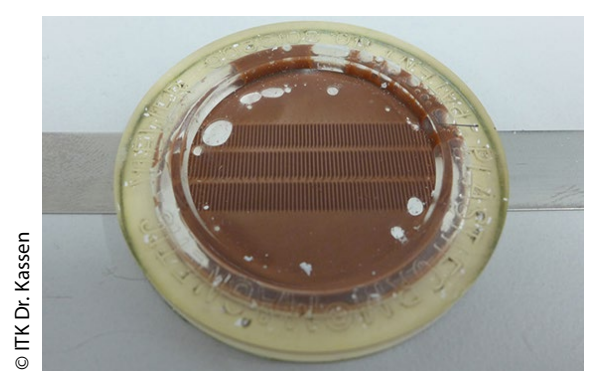

Magnetisch beschichtetes Band mit aufgebrachter Codierung. definierte magnetische Struktur beziehungsweise Funktionsschicht, die sich mit einem Signalmuster codieren und per Sensor auslesen lässt, um eine Positionsbestimmung vornehmen zu können. „Im Zusammenspiel mit den integrierten Sensoren, welche die Signale auslesen, ermöglichen unsere Schichten das Anfahren von Positionen bis auf fünf Nanometer genau“, erläutert Dr. Bandorf. Die Tische erlauben durch das integrierte Messsystem eine Absolutbestimmung der Position ohne Referenzierung. Wiederholgenauigkeiten von $\pm 100 \mathrm{~nm}$ sind erreichbar. Dies ist inbesondere bei der Untersuchung von lebenden Objekten wichtig, wo die Untersuchungszeit oftmals knapp und ein schnelles Positionieren daher essentiell ist.

\section{Umweltfreundliches Beschichtungsverfahren}

Die CoSm-Schichten ersetzen galvanische Kobaltschichten, für die umweltschädliche Chemikalien benötigt werden. Sie werden mit dem am Fraunhofer IST entwickelten Vakuumbeschichtungsverfahren Hohlkathoden-Gasfluss-Sputtern hergestellt. Anders als bei galvanischen Verfahren kommen hier keine Schadstoffe zum Einsatz. Die Schichten zeichnen sich durch ihre Robustheit und Langlebigkeit sowie durch besonders gute magnetische Eigenschaften aus: Sie ermöglichen ein stärkeres magne- tisches Signal und berührungsloses Messen. Zudem lassen sich auch Messungen in geschlossenen Bauteilen wie etwa Hydraulikzylindern vornehmen, an die optische Systeme nicht gelangen.

Anders als reine Kobaltschichten sind CoSm-Schichten nicht so leicht ummagnetisierbar, sie sind unempfindlich gegenüber Störfeldern und erlauben das Messen in verschmutzten Bereichen. Außerdem lassen sich sehr feine Schichtdicken erzielen. Winkelpositionen und Radialbewegungen können ebenfalls gemessen werden, was insbesondere für Robotikanwendungen, zum Beispiel in der Automobilindustrie, relevant ist. „Beim Aufbringen einer kompakten CoSm-Schicht direkt auf das Bauteil, beispielsweise einem Kugellager, erhält man zusätzliche Informationen“, so Dr. Bandorf. Auch im Bereich der Elektromobilität steigt die Nachfrage nach hochgenauen magnetischen Messsystemen. //

\section{Kontakt}

Fraunhofer-Institut für Schicht- und Oberflächentechnik IST

Braunschweig

Dr. Simone Kondruweit

www.fraunhofer.de 